\title{
Topical Issue on Advances in Computational Methods for Soft Matter Systems
}

Published online: 29 August 2018 - C EDP Sciences / Società Italiana di Fisica / Springer-Verlag GmbH Germany, part of Springer Nature, 2018

We like to think of scientific articles as stories, and this Topical Issue is a collection of some of the best stories in the computational Soft Matter genre. As a field, Soft Matter is rapidly evolving: at the beginning the protagonists used to belong to one of three archetypes (the colloid, the polymer, and the amphiphilic molecule), but recently many characters have entered the scene, from artificial nanoparticles, to complex biological molecules, and active materials. These stories are usually set in a world between $10 \mathrm{~nm}$ to $10 \mu \mathrm{m}$, and all the events last anywhere between a few nanoseconds up to the time you can measure with your watch. It is a strange stage down there: the interactions between the protagonists are always affected by large fluctuations, leaving everything permanently disordered and continuously changing. The motions are often overdamped, and nothing occurs just because of inertia. Many of the characters are very susceptible to the environment around them, and act accordingly. For instance, colloidal particles do not usually like each other; however, if the room fills with polymers, they will happily decide to get together. Sometimes these bonds are irreversible, and the story stops before reaching its natural conclusion. If there is a lot of drama between two subjects, amphiphiles will always help reducing the tension. Some protagonists have super-powers, from reduced valence interactions, to self-propelling abilities, to flying colloidal carpets.

As writers of these stories, how can we make sense of what is happening in this small world? Luckily for us, as the protagonists grew in number and originality, and the phenomena became more and more complex, also our language has adapted. Here, the language we are interested in is that of computer simulations. Among the tools available to a Soft Matter scientist, probably nothing has progressed so quickly and so drastically as computer simulations, propelled by the exponential growth in computing power, and a never ending stream of new techniques and clever algorithms. This Topical Issue, Advances in Computational Methods for Soft Matter Systems, published in The European Physical Journal $E$, is a collection of articles that showcase the state of the art in computer simulations across the field of Soft Matter. Every article features a computational insight that has led to the solution of an interesting story in Soft Matter.

We are very proud of the quality and range of contributions to the Topical Issue. Together they comprise a wide selection of topics, that showcase some of the best methods that have emerged to tackle the related computational challenges.

Some of the biggest problems in the simulation of Soft Matter systems relate to the long time-scales required to observe complex relaxation phenomena. The prototypical example of a rapid dynamical slowing down is given by glassformers. Anomalous dynamical properties are often observed in glasses, where relaxation times span several orders of magnitude with a small change in temperature. The article of Daniele Coslovich et al., shows how the use of multi-CPU and multi-GPU parallel tempering techniques enables the study of the crossover in the fluctuations of the dynamics of a glass-forming mixture, which would not be otherwise possible with conventional methods [1]. The origin of dynamic heterogeneities in another glass-forming mixture is investigated via a large-deviation theory and extended ensemble techniques in the article by Turci et al., which hints at the presence of a dynamic phase transition between an active and an inactive phase, distinguished by the concentration of local icosahedral structures [2]. Harnessing the power of modern computing systems to greatly reduce the wall-clock time, with only a modest increase of computational effort, is at the heart of the swarm relaxation technique by Malek et al. [3], in which the usual time averages in molecular dynamics simulations are replaced with ensemble averages obtained from independent runs.

Activated processes present computational challenges associated with both overcoming large free-energy barriers, and determining the correct reaction coordinate for the transition. The article by Tinti et al. [4] shows how the string method in collective variables can be used to solve a very challenging problem such as the vapor nucleation in lyophobic nanopores. By looking at the most probable nucleation pathway in a high-dimensional free-energy landscape, 
Tinti et al. show the different free-energy costs associated with both symmetric and asymmetric transition paths. They find that asymmetric bubble formation is significantly more likely than the symmetric case, and support their analysis with a continuum model.

Another area of Soft Matter which is becoming increasingly more important is the study of active materials, and in particular of microscopic swimmers. For these systems, the biggest difficulty comes from the need to accurately model the hydrodynamic interactions, which greatly increases the computational cost of the simulations. A brilliant solution to this problem is found in the article by Yoshinaga et al. [5], where a new numerical scheme is developed which allows for the simulations of a large number of active particles that have accurate hydrodynamic interactions even when close to each other. The article shows that lubrication forces, often ignored in other methods, are in competition with long-range hydrodynamic forces, and can completely change the behaviour of swimmers. The contribution of Zöttl and Stark provides a thorough discussion on modelling squirmers - simple spherical model swimmers - by explicitly taking into account the effect of the solvent with the multi-particle collision dynamics (MPCD) method [6]. Martinez et al. investigate the behaviour of a two-dimensional system of Vicseck hard disks in the presence of obstacles. In particular, the authors study the collective behaviour of these particles with two different bouncing rules and in the presence of moving and fixed obstacles [7]. Even when the system under study is not active, non-equilibrium simulations can still pose a formidable challenge, and choosing the right methods and parameters can be key. RuizFranco and coworkers present a careful study of the effects of using different thermostats in dynamical simulations of implicit-solvent systems under shear. This contribution will be useful to anyone approaching non-equilibrium molecular dynamics simulations [8].

The world of (bio)polymers is vast, dark and dangerous. From a computational perspective, the topological constraints that are often present make macromolecular systems formidable systems to simulate. Some of the difficulties that are encountered can be alleviated by using the right models and tools. The first of the two papers by Tubiana and collaborators introduces KymoKnot, a software package and webserver that analyses polymers configurations, generated from simulations or extracted from, for instance, the PDB database, to identify and locate physical and proper knots [9]. In the second paper by Tubiana et al., a method to implement efficient concerted rotations, which can greatly speed up the simulation of, for instance, proteins, is presented [10]. In the last article that deals with macromolecules, Henrich et al. describe the implementation of oxDNA, a popular coarse-grained DNA model, into LAMMPS [11]. The article, in addition to discussing the model implementation and its parallel performance, also contains a tutorial section that shows how to set up a simple simulation.

The behaviour of systems composed of particles interacting through anisotropic interactions is a central topic in modern soft matter science. Together with experimental advances, the last years have seen a flourishing of new simulation models and techniques tailored to the investigation of complex building blocks. In their contribution, Minina et al. focus on the interplay between thermodynamics (and, in particular, the compressibility) and the microstructure of the paradigmatic dipolar soft sphere model [12]. Going from dipoles to charges, the paper by Ferrari et al. describes in a detailed fashion a numerical algorithm for efficiently simulating inverse patchy colloids, e.g. particles with differently charged surface regions [13]. On a similar note, Orellana et al. delve into the topic of speeding-up Monte Carlo simulations of hard patchy cylinders, which are often used as toy models for liquid crystal phases, focussing on improving the algorithm devoted to checking overlaps between the particles [14]. Finally, the Colloquium paper by Rovigatti et al. is a useful tool for all those students and early-career researchers who want to learn how to simulate the so-called patchy particles [15]. The contribution is complemented by an educational code written in the C language.

Coarse-grained descriptions of complex systems can be used to look at phenomena that occur at time- and lengthscales that would not be otherwise accessible. However, it is not always straightforward to draw a comparison between the original and the coarse-grained description. Heidari et al. present a detailed study of the practical and technical aspects of a simulation technique that couples two different models of the same system (usually, one much coarser than the other) in the same simulation. This technique allows to extract quantitative information on the differences between the two models, and is a very promising tool to investigate the properties of liquids and solids [16]. On a related note, Munaò et al. present a detailed study on coarse-graining systems of nanoparticles of different nature. After calibrating the method against silica nanoparticles, they compute the potential of mean force between bare and polymer-coated gold nanoparticles [17].

We hope that you will enjoy these stories as much as we did, and that you will find in here the inspiration for writing your next Soft Matter story. 


\section{References}

1. Daniele Coslovich, Misaki Ozawa, Walter Kob, Eur. Phys. J. E 41, 62 (2018).

2. Francesco Turci, Thomas Speck, C. Patrick Royall, Eur. Phys. J. E 41, 54 (2018).

3. Shahrazad M.A. Malek, Richard K. Bowles, Ivan Saika-Voivod, Francesco Sciortino, Peter H. Poole, Eur. Phys. J. E 40, 98 (2017).

4. Antonio Tinti, Alberto Giacomello, Carlo Massimo Casciola, Eur. Phys. J. E 41, 52 (2018).

5. Natsuhiki Yoshinaga, Tanniemola B. Liverpool, Eur. Phys. J. E 41, 76 (2018).

6. Andreas Zöttl, Holger Stark, Eur. Phys. J. E 41, 61 (2018).

7. Raul Martinez, Francisco Alarcon, Diego Rogel Rodriguez, Juan Luis Aragones, Chantal Valeriani, Eur. Phys. J. E 41, 91 (2018).

8. José Ruiz-Franco, Lorenzo Rovigatti, Emanuela Zaccarelli, Eur. Phys. J. E 41, 80 (2018).

9. Luca Tubiana, Guido Polles, Enzo Orlandini, Cristian Micheletti, Eur. Phys. J. E 41, 72 (2018).

10. Luca Tubiana, Miroslav Jurásek, Ivan Coluzza, Eur. Phys. J. E 41, 87 (2018).

11. Oliver Henrich, Yair Augusto Gutiérrez Fosado, Tine Curk, Thomas E. Ouldridge, Eur. Phys. J. E 41, 57 (2018).

12. Elena S. Minina, Elena S. Pyanzina, Ekaterina V. Novak, Sofia S. Kantorovich, Eur. Phys. J. E 41, 67 (2018)

13. Silvano Ferrari, Gerhard Kahl, Emanuela Bianchi, Eur. Phys. J. E 41, 43 (2018).

14. Alberto Giacomo Orellana, Emanuele Romani, Cristiano De Michele, Eur. Phys. J. E 41, 51 (2018).

15. Lorenzo Rovigatti, John Russo, Flavio Romano, Eur. Phys. J. E 41, 59 (2018).

16. Maziar Heidari, Robinson Cortes-Huerto, Kurt Kremer, Raffaello Potestio, Eur. Phys. J. E 41, 64 (2018).

17. Gianmarco Munaò, Andrea Correa, Antonio Pizzirusso, Giuseppe Milano, Eur. Phys. J. E 41, 38 (2018). 